\title{
QED: Chiral transition and the issue of triviality
}

\author{
Aleksandar Kocić \\ University of Illinois at Urbana-Champaign, Urbana-IL 61801
}

I give a review and progress report on studies of lattice QED. I emphasize analytical results and methods that are applied in data analysis. Also, I derive some bounds for the critical exponents and establish their connection with scaling violations. Triviality, as realized in $\phi^{4}$ theory, is ruled out on theoretical grounds. I show that the present data, if analyzed correctly, all lead to the same conclusions. They are compatible with power law scaling with nongaussian exponents.

\section{Introduction}

The most important theoretical issue related to QED is the question of its existence and the nature of the continuum limit. Traditional wisdom is that non-asymptotically theories do not have an interacting continuum limit in four dimension. This belief has been based on perturbation theory and, until recently, hasn't been challenged outside of the perturbative context. The study of lattice QED is an atempt to clarify this issue [1] [2] 34] [-

The central problem in the study of the continuum limit of a theory is the verification of hyperscaling [5]. The starting point is the free energy from which all connected correlation functions can be generated: $F_{\text {sing }}=t^{2-\alpha} F\left(h / t^{\Delta}\right)$. Having the dimension of inverse volume, hyperscaling implies that $F_{\text {sing }} \sim \xi^{-d}$. The renormalized coupling is defined as

$$
g_{R}=-\frac{\chi^{(n l)}}{\chi^{2} \xi^{d}}
$$

where $\chi^{(n l)}=\partial^{3} M / \partial h^{3}$ is the zero-momentum projection of the connected four-point function. If the action is gaussian, Wick's theorem applies and the $N$-point functions factorize. Thus, for a gaussian theory $g_{R}$ vanishes and is non-zero otherwise. Using the hyperscaling hypothesis, eq.(1.1) can be converted into

$$
g_{R} \sim \xi^{(2 \Delta-\gamma-d \nu) / \nu}
$$

Being dimensionless, $g_{R}$ should be independent of $\xi$ if $\xi$ is the only scale. Thus, the validity of hyperscaling requires that the exponent must vanish. So, hyperscaling implies the relation, $2 \Delta-\gamma-d \nu=0$, between the critical indices. It is known in general that the following inequality [6] holds $2 \Delta \leq \gamma+d \nu$. This means that the exponent in the expression for $g_{R}$ is always nonpositive, so that violations of hyperscaling imply that the resulting theory is non-interacting. Above four dimensions, the exponents are gaus$\operatorname{sian}(\gamma=1, \Delta=3 / 2, \nu=1 / 2)$. In this case, it is easy to verify the above inequality - it amounts to $d \geq 4$. In four dimensions most field theoretical models critical exponents have the mean-field values, but with logarithmic corections that drive $g_{R}$ to zero. From here, the importance of the knowledge of critical exponents becomes clear. Establishing their non-gaussian values would be a significant step towads ruling out the triviality of the theory.

The recent discovery that non-compact $Q E D$ undergoes a second order phase transition [1] from a massless to a massive phase with spontaneously broken chiral symmetry reopened the old question of the existence of quantum electrodynamics. In what follows I will survey what has been done.

When studying chiral symmetry breaking in gauge theories one is faced with the following problem. Because of the singular nature of the chiral condensate, the chiral limit is not directly accessible in numerical simulations and work at finite bare mass is required. In this way the precise position of the critical coupling is difficult to determine. 
Figure 1. Mass ratio versus bare mass for $N=2$ theory on a $16^{4}$ lattice. Solid lines are the fits obtained from the EOS.

A usual way to procede is to commit oneself to some scenario and extrapolate to the chiral limit looking for the best fit etc.. The index $\delta$ determines the approach to the chiral limit. It is defined at the critical point by $m \sim<\bar{\psi} \psi>^{\delta},(t=$ $0)$. Here, I will discuss an alternative to the traditional approach to finding the critical exponents in a given theory and show how it is applied to $Q E D$ where the traditional approach led to ambiguous and controversial results in the past.

Consider the effect of chiral symmetry breaking on the meson spectrum from a physical point of view. In the symmetric phase, chiral symmetry requires the degeneracy between the chiral partners. Therefore, in the chiral limit the ratio $R=M_{\pi}^{2} / M_{\sigma}^{2}=1$. As the bare mass increases, the ratio decreases. In the broken phase, however, the ratio vanishes in the chiral limit because the pion is a Goldstone boson. Behavior of the mass ratio is displayed in Fig.1. The data are taken from [9].

The connection between the mass ratio and the order parameter is obtained from the equation of state (EOS).

$$
\begin{gathered}
h_{a}=M_{a} M^{\delta-1} f\left(\frac{t}{M^{1 / \beta}}\right) \\
\frac{\chi_{\sigma}^{-1}}{\chi_{\pi}^{-1}}=\delta-\frac{x}{\beta} \frac{f^{\prime}(x)}{f(x)}
\end{gathered}
$$

At the critical point eq.(1.4) results in a simple equation

$$
\frac{\chi_{L}^{-1}}{\chi_{T}^{-1}}=\delta, \quad t=0
$$

The ratio is independent of the symmetry breaking field! The knowledge of the mass ratio can be used to determine both the critical point and exponent $\delta$ [8].

\section{Two and Four Flavor Theory}

I first discuss the four flavor data. Simulations in this case were done on smaller lattices $\left(12^{4}\right.$ by DESY [7] and $10^{4}$ by Illinois group [10]). For $N=2$ theory simulations were done on a bigger lattice $\left(16^{4}\right)$ with better statistics by the Illinois group [9]. The quality of the four flavor data is much more modest and are contaminated by the finite size effects. This being the case, the conclusions that have been drawn in the past are to be taken with caution.

Figure 2. Mass ratio data versus $m$ taken from the DESY data. The simbols are explained in the text.

The plot of mass ratios versus the bare mass 
is shown in Fig.2. The data are taken from the DESY group [7]. Critical parameters from these data are extracted simply. They are $\beta_{c}=$ $0.205, \delta=2.3$. The order parameter data, are shown in Fig.3. The data on this plot are from the Illinois group [10]. They are consistent with those of the DESY group. $m=0.03$ was the lowest mass that was insensitive to the finiteness of the lattice [10]. Thus, unlike in the analysis of the DESY group, $m=0.01$ and 0.02 are not included here. Both, $\beta_{c}$ and $\delta$ obtained this way agree with the values extracted from the mass ratios 10]. It should be noted that the critical coupling proposed by the DESY group [7], $\beta_{c}=0.185$ (dashed line), lies deeply in the strong coupling region. Both mass ratio and Fig. 3 show this very clearly. No extrapolations or additional assumptions were made so far. Note that if $\beta_{c}$ were indeed 0.185 , as proposed by the DESY group, then the corresponding isotherm would have to bend upwards. Clearly, such a curvature would correspond to $\langle\bar{\psi} \psi>$ that is too small for a given value of $m$, an effect typical for symmetry breaking in a small volume. In the thermodynamic limit, however, this is not possible on theoretical grounds as I will discuss later.

Figure 3. Order parameter data for the four flavor theory.

The EOS (Fig.4) results in a straight line fit to $f(x)$ and exponent $\beta=0.764$. This EOS gives predictions for the mass ratio data and the fit is given by the solid lines in Fig.2. The fit is compelling and we conclude that consistency is satisfactory.

Figure 4. EOS fit to power-law scaling for $\mathrm{N}=4$ data from Illinois gruop.

Now, I review briefly the atempts of the DESY group to fit the same data to the logarithmically improved mean field theory. In ref.[7] a logarithmically improved $O(2)$ sigma model is used for fitting purposes and it fits the data very well. The EOS reads,

$$
m=\tau \frac{\sigma}{\ln ^{p}\left|\sigma^{-1}\right|}+\theta \frac{\sigma^{3}}{\ln \left|\sigma^{-1}\right|}
$$

where $\sigma=\langle\bar{\psi} \psi\rangle, \tau=\tau_{1} \theta\left(1-\beta / \beta_{c}\right)$ and $\theta^{-1}=$ $\theta_{o}+\theta_{1}\left(1-\beta / \beta_{c}\right)$. Choosing specific values for the five parameters $\beta_{c}, p, \tau_{1}, \theta_{c}$ and $\theta_{1}$, a very good fit to the data is found, Fig.5. The resulting chiral transition occurs at $\beta_{c}=0.186(1)$ with mean field critical indices built in. Since the fit involves five parameters, its significance is certainly debatable. Let us subject eq.(2.1) to the same test that the power-law scaling fit has just passed. In particular, from Sec. 8 of ref.[7] we read off a formula for $R=M_{\pi}^{2} / M_{\sigma}^{2}$ calculated in the logarithmically-improved $O(2)$ sigma model. This is done in Fig.6. Obviously, the fit fails qualitatively. 
Figure 5. EOS to MF+log scaling, eq.(2.1). DESY data.

What is wrong with the MF+log scenario? It appears to fit the order parameter very well, but it fails with the masses. The answer is simple. Logarithmic violations of scaling as displayed in eq.(2.1) can never appear in the case of chiral phase transition. At the critical point, eq.(2.1) predicts

$$
m \sim \frac{<\bar{\psi} \psi>^{3}}{\log (1 /<\bar{\psi} \psi>)}
$$

Because of the scaling violations, the RHS vanishes faster then a pure power. So the "effective" $\delta$ is bigger then its mean-field value. In the Nambu-Jona-Lasinio model for example critical EOS gives, on the other hand,

$$
m \sim<\bar{\psi} \psi>^{3} \log (1 /<\bar{\psi} \psi>)
$$

Unlike for magnets, the log's appear in the numerator - the RHS vanishes slower then the pure power and the "effective" $\delta$ is smaller then the (pure) mean-field value. As shown in [12], this difference in the position of the logarithm i.e. the sign of the scaling violations is generic for the two models. It is independent of the approximation and follows from the basic differences in physics of the two systems. In [12] it was shown that exponent $\delta$ satisfies two different bounds, depending on wheher Goldstone bosons are elementary or composit. For magnets $\delta>3$, while for fermions
Figure 6. Mass ratio data versus the prediction from the MF+log EOS. Desy data

$\delta<3$. These bounds are respected by the logarithmic violation of scaling in four dimensions [12]. The origin of these bounds on $\delta$ lies in the fact that for magnets weak coupling phase is broken wheras for fermions the situation is reversed. Thus, the failure of the fits of ref. [7] is understandable and expected since it is borrowed from the magnetic context and applied to the fermions. The source of errors in the fits of ref.[7] is missidentifiacion of the physics and the lack of control of finite size effects. Because of the small $\left(12^{4}\right)$ lattice, $\beta=0.185$ that was mistakenly identified with the critical coupling by the DESY group was deep at the strong coupling phase. Such a misidentification coresponds to a smaller size of the strong coupling phase and the larger value of $\delta$.

Concerning the other results reported by the DESY group in [7], several comments are in order. Some preliminary attempts to extract renormalized charge and fermion mass have been made in ref.[7]. I should mention some obvious technical problems that concern the state of affairs related to this attempt. Like order parameter studies, simulations of the renormalized charge and fermion mass were done on a $12^{4}$ lattice. The procedure that was employed assumed the masslessness of the photon. The renormalized charge was then extracted as the residue of the photon prop- 
agator evaluated at zero momentum. However, due to the finite volume constraint, the propagator was evaluated at finite momentum and the result was extrapolated to $k=0$. This is a mysterious step since ref. [7] does not make any attempt to clarify the origin of the extrapolation procedure and its stability to a change in volume.

In fact, this size of the lattice was too small to yield qualitatively correct results for the quantities like $\langle\bar{\psi} \psi>$ and meson masses. These data were seriously distorted by the finiteness of the volume. The photons, being presumably massless, can only be more sensitive to such a small volume. The same holds for the fermion mass since fermions are charged and are, in principle, much more sensitive to the presence of the massless photons then are mesons. Therefore, none of the results and the conclusions regarding the photon and fermion propagator, can be taken seriously. The quality of the fermion mass and renormalized charge data is not good to yield any quantitative statements and, if taken seriously, they lead to incorrect and inconsistent result.

For example, consider the fermion mass data taken from the DESY group. In general, for any mass, $M_{A}$, in the scaling region the following equation holds: $M_{A}=t^{\nu} G_{A}\left(m / t^{\Delta}\right)$. Thus, the ratio $M_{F} / M_{\sigma}=G\left(m / t^{\Delta}\right)$ is a function of only one variable. This is true if both masses scale. The data for $M_{F} / M_{\sigma}$, taken from [7], are shown in Fig.7. Regardless of the value of $m$, the ratio at the critical point, $t=0$, always has the same value. Thus, the curves $R$ vs $\beta$ have to cross at $\beta_{c}$ (insert of Fig.7). However, data show that the curves never cross! The fermion mass, as taken from the data, does not scale. It doesn't show proper restoration of chiral symmetry probably because finite volume effects keeps it too heavy. Clearly, if scaling properties were to be recovered, the extrapolation would drive us to the weaker coupling in order to reduce the fermion mass to its value compatible with the phase of restored symmetry. This is why the conclusions obtained through extrapolation of the data in ref. [7] agreeed so well with renormalized perturbation theory - high fermion mass was confused for low renormalized charge. This is how the authors of ref. [7 misinterpreted their data as supporting the zero charge continuum limit.

Figure 7. Fermion-sigma mass ratio versus bare coupling. DESY data. The insert is an illustration of the behavior of mass ratio in the scaling region.

As far as the two flavor data is concerned, the extensive studies have been done on lattices ranging from $10^{4}$ to $16^{4}$. The results have been reported in ref. [9]. The same strategy has been applied as in the case of four flavor theory. In Fig.1. the mass ratio data are confronted with the predictions of the power-law EOS (solid lines).

A different approach, using microcanonical method, is adopted by the Zaragoza group [4]. The results for the critical coupling and the critical indices obtained by the Illinois and Zaragoza group agree.

A brief comment about perturbation theory should be made. The applicability of perturbation theory in the case of scalar theories has been diagnosed early on. For example, in the $\sigma$-model the weak coupling phase is at low-temperatures. Due to the presence of Goldstone bosons, all the correlation functions are saturated with the massles states and the entire low-temperature phase is massless. Every point is a critical point in the limit of vanishing magnetic field. Thus, the low temperature expansion is an expansion in powers of $T$. The terms of the form $\exp (-M / T)$ 
are absent and there is no danger that they will be omitted by using perturbation theory. In this way, in principle, the critical region can be accessed through perturbation theory which is quite accurate close to two dimensions because the critical temperature moves to the origin. Clearly such reasoning can not be applied to fermions simply because the weak coupling phase is symmetric. Thus, no matter how small the coupling is, perturbation theory omits the Goldstone physics as a matter of principle. It can not produce bound states that accompany the chiral transition and its use is questionable in this case.

This holds for $Q E D$ as well although renormalized charge is bounded from above [11]. In fact, it is difficult to see how perturbation theory could communicate with the chiral transition. For example, the fact that the value of the mass ratio at the critical point is independent of the bare mass is a truely nonperturbative result and could not be obtained from any expansion in powers of $\alpha_{R}$. Since $\alpha_{R}$ is dimensionless, at the critical point it must be independent of $m$ as well, which is clearly impossible in perturbation theory where screening is sensitive to the fermion mass. In other words, conflicting RG trajectories must appear if perturbative analysis of the renormalized charge is used.

\section{Conclusions}

We have seen so far that the problem of analyzing the scaling region of an unknown theory is not straightforward. Depending on the accuracy of the data, ambiguous results can follow. If $\beta_{c}$ is underestimated, and appropriate extrapolations done, the "best fit" is given by mean field exponents supplemented with the log's in the wrong place! We have been able to eliminate this possibility on theoretical grounds 12 . At this stage it is fair to say only that the data support the power-law scaling with non-gaussian exponents. The data of all three groups 10] [7] agree on this point. Triviality as realized in $\phi^{4}$ theory is definitely ruled out. The scenario proposed by the DESY group [7] is dismissed on theoretical grounds, without a need to consult the data. It remains to be seen whether the power law scal- ing with $\delta \approx 2.3$ can be distinguished numerically from mean-field plus log's with the log's as in Nambu-Jona-Lasinio model. This is the question that needs to be addressed eventually since the value of $\delta$ obtained from the simulations and is not too far from its mean-field value. Work on this problem is in progress.

\section{Acknowledgement}

Most of the work reported here was done in collaboration with John Kogut and Maria-Paola Lombardo. I wish to acknowledge the discussions with V. Azcoiti, E. Fradkin, P. Hasenfratz, E. Seiler and P. Weisz. This work is supported by NSF-PHY 92-00148.

\section{REFERENCES}

1. E. Dagotto, A. Kocić and J. B. Kogut, Phys. Rev. Lett.. 60, 772 (1988); 61, 2416 (1988).

2. A. M. Horowitz, Phys.Lett. B219 (1989) 329.

3. M. Gockeler, et al., Nuc. Phys. B334, 527 (1990).

4. A. Azcoiti, G. Di Carlo and A. F. Grillo, Int. Jour. Mod. Phys. A8, 2239 (1993).

5. M. Aizenman, Comm. Math. Phys. 86, 1 (1982); C. Aragao de Carvalho, S. Caracciolo and J. Frohlich, Nucl. Phys. 215[FS7], 209 (1983).

6. B. D. Josephson, Proc. Phys. Soc. 92 (1967) 269, 276.

7. M. Gockeler, et al., Nuc. Phys. B371, 713 (1992).

8. A. Kocić, J. B. Kogut and M.-P. Lombardo, Nuc. Phys. B398, 376 (1993).

9. S. J. Hands, et al., CERN-TH 6609/92.

10. A. Kocić, J. B. Kogut and K. C. Wang, Nucl. Phys. B398 (1993) 405.

11. M. Lüscher, Nucl. Phys. B341, 341 (1990).

12. A. Kocić and J. B. Kogut, Compositness, Triviality and Bounds on Critical Exponents for Fermions and magnets, Illinois preprint. 